doi:10.1017/S0022215116003479

Free Papers (F763)

\section{ID: 763.4}

Surgical treatment of cholesteatoma:

Criteria for the selection of the surgical technique

Presenting Author: Alejandro Harguindey AntolíCandela

Alejandro Harguindey Antolí-Candela ${ }^{1}$, Francisco J. Olarieta Soto ${ }^{2}$, Francisco Antoli-Candela Cano ${ }^{2}$

${ }^{1}$ Instituto Otorrinolaringologico De Madrid (IOM), ${ }^{2}$ Instituto Otorrinolaringológico de Madrid (IOM)

\section{Learning Objectives:}

Introduction: The surgical technique in cholesteatoma is decided on the bases of the following criteria:

1.- Anatomical characteristics of the mastoid.

2.- Clinical type of cholesteatoma:

Congenital.

Primary acquired.

Secondary acquired.

3.- Surgical type of cholesteatoma:

Encapsulated and non-encapsulated.

4.- The estate of the mucose membrane.

Absent, cholesterol granuloma, polyps in the middle ear, etc....

5.- Extension of the cholesteatoma.

Material and method: The above described criteria is revised in 380 consecutively operated. The different techniques and their results are described.

At present the most frequent technique is Intact Canal Wall tympanoplasty (210 ICW, 60\%) followed by different types of Canal Wall Down (140 CWD 40\%) tympanoplasties. In $350(92 \%)$ a tympanoplasty was performed.

The incidence of Radical Mastoidectomies (RM), middle ear exclusion (MEE) and Subtotal petrosectomies (STP) are very infrequent $(8 \%)$ and are performed mostly in revision surgery in cases with a failure of CWD tympanoplasty.

Results: The failures are subdivided in recurrent and residual cholesteatoma:

- We have five percent of recurrent cholesteatoma in the adult and eight percent in children.

- In $20 \%$ of the cases there is a residual cholesteatoma. Only $2 \%$ of them in CWD tympanoplasty, RM, MEE or STP.

The hearing results can be summarized saying that in $60 \%$ of the patients there is useful hearing without the need for a hearing aid. These results are closely related to the technique used for surgery.

Conclusions: Adequate selection of the surgical technique for each individual case is the first goal to obtain best results both to minimize recurrences and to obtain the best possible hearing results.

There is not a surgical technique that is best for all cases.

Diffusion MRI is very useful to follow cases of MEE and STP and has made us to indicate these techniques more often.

doi:10.1017/S0022215116003480

\section{Free Papers (F763)}

\section{ID: 763.5}

Functional results and quality of life in patients undergoing surgery for cholesteatoma: Canal Wall Down versus Canal Wall Up technique

Presenting Author: Bruno Sergi

Bruno Sergi, Daniela Lucidi

Catholic University School of Medicine and Surgery, Rome, Italy

Learning Objectives: Canal Wall Down Mastoidectomy has traditionally been associated with a poorer Quality of Life, based on the limitations and restrictions resulting from the wide neo-mastoid cavity. However, according to our results, a substantial difference in the subjective perception of the post-operative outcomes between CWD and CWU should not be taken for granted.

Introduction: The benefits and disadvantages of Canal Wall Up (CWU) versus Canal Wall Down (CWD) mastoidectomy in cholesteatoma surgery have been debated for decades: comparison is mostly based on auditory results and recurrence rate. However, the most common complaint among cholesteatoma patients is daily social impediment (ear discharge, water restriction, pain, doctor visits). The aim of our study was to assess the quality of life (QOL) and hearing function in CWD vs. CWU technique.

Methods: We enrolled 81 patients subjected to mastoidectomy for cholesteatoma, at Department of Head and Neck Surgery, Catholic University, Rome (Jan 2010-Dec 2013). In 50 patients a CWD technique, without "obliteration" of the mastoid cavity, was performed (Group A) whereas 31 patients underwent a CWU technique. Pure tone audiometry (PTA) was performed 24 hours pre- and 12 months postoperatively. Chronic Ear Survey (CES) was administered 3 months after surgery. Twelve months after surgery patients were subjected to re-administration of CES together with a second questionnaire: Chronic Otitis Media Outcome Test15 (COMOT-15).

Results: The difference between mean postoperative PTA in Group A and Group B was statistically significant (respectively $72 \mathrm{~dB}$ vs. $45 \mathrm{~dB} ; \mathrm{p}<0.05$ ). The mean CES scores were not significantly different between groups $(\mathrm{p}<0.05)$, except for the subscale "Symptoms". Also, mean COMOT-15 results didn't not significantly 